\title{
Peridynamic Modeling of plain concrete structures under monotonic loading
}

\author{
Jiezhi Lu ${ }^{1, a}$, Yaoting Zhang, b, Zhijun Chen ${ }^{1}$ \\ ${ }^{1}$ School of Civil Engineering \& Mechanics, Huazhong University of Science and Technology, \\ Wuhan, 430074, China

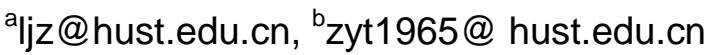

Keywords: Peridynamic, Plain concrete structures, Monotonic loading, Crack propagation

\begin{abstract}
This study presents an application of the peridynamics method to the analysis of concrete structures, the quasi-static fracture behavior of concrete column is simulated with the proposed model, numerical result shows that the proposed peridynamic model can predict both the progressive failure process and the ultimately capacity of concrete structures with reasonable accuracy.
\end{abstract}

\section{Introduction}

Concrete structures are widely used in the field of civil engineering, which is found to be suffering from deterioration of durability involves the progressive process of micro-cracking, delamination and other irreversible internal damage, especially in severe environmental conditions. Consequently, many construction projects lost their function prematurely and even collapse during the prescriptive service period. Therefore, the numerical modeling of progressive damage in concrete structures has become a major concern and attracted extensive research interest in the past decades. Different constitutive models and numerical approaches have been adopted to handling with complicated fracture problems, However, most methods still suffer the poor efficiency and unsatisfactory simulation accuracy when dealing with discontinuous or singularities [1].

In an attempt to overcome the aforementioned difficulties, Silling [2, 3] from Sandia National Laboratory reformulated the basic equation of continuum mechanics, and then put forward a special nonlocal theory known as peridynamics, in which no assumptions are required regarding continuity of deformation. Particularly, bonds, which contains constitutive information of the material, were used to reproduce the nonlocal interacting forces between particles over certain distance. Moreover, In contrast to the partial differential equations used in the classical formulation, this theory instead uses spatial integral equations which permits the cracks spontaneously occur at multiple sites and freely extend along arbitrary path without the need for special remedial techniques. Therefore, the peridynamic theory is very suitable for predicting the progressive failure process and final failure modes of various materials.

Numerous articles on the application and evolution of the bond-based peridynamic model appears in the literature. Gerstle et al. [4] developed several models for concrete with micro-elastic stiffness c and a cut-off stretch s*, and the latest one proved to be more accurately behavior in both tension and compression regime. Huang et al. [5] proposed new numerical algorithms for quasi-static bond-based PD simulation including local damping, step-by-step loading, and a non-equilibrium criterion. However, for the current peridynamic research efforts on the concrete structure, the fracture behavior of concrete structure are usually simulated through the breakage of bonds which are determined by some specified stretch related to the fracture energy, while the fracture energy may differs in the same concrete specimen. In this paper, a new peridynamic model for concrete structures was proposed. Based on the proposed peridynamic model, the detailed process of progressive damage and final failure mode in underpinned concrete column was analyzed and compared with the experimental observations. 


\section{Bond-based Constitutive Model for Concrete}

The constitutive model is defined through the relationship between bond stretch and pairwise force among material particles, which means that the material damage is introduced at the bond-level. Silling et al. [2] introduced an original constitutive model for quasi brittle materials which associated the critical stretch $s_{0}$ with the fracture energy $G_{F}$, the bond would break when the elongation reached $s_{0}$. Gerstle et al. [4] correlated the critical stretch $s_{0}$ with the uniaxial tensile $f_{t}$ and uniaxial compressive strength $f_{c}$, and developed a eight-parameters micropolar peridynamic model for concrete, In the present work, the constitutive model is simplified as depicted in Fig. 1.

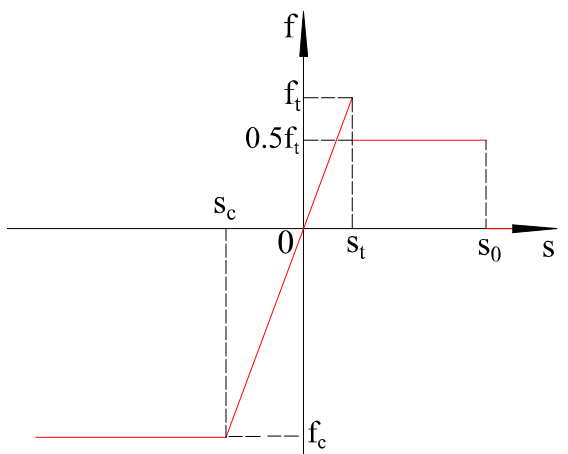

Fig. 1 Constitutive model for concrete.

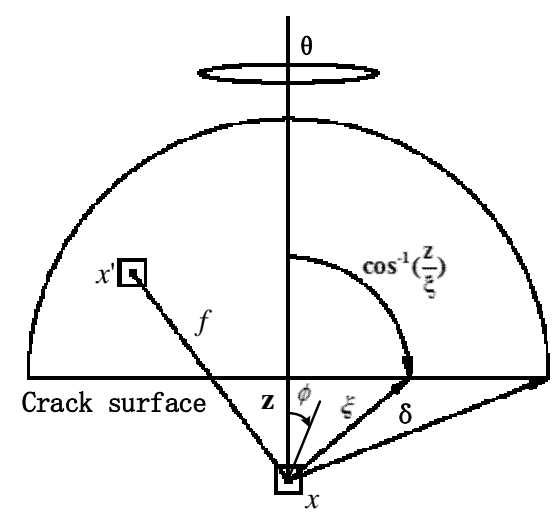

Fig. 2 Evaluation of fracture energy.

the fracture energy $G_{F}$ required to break all the bonds per unit fracture area can be derived as in [2]:

$$
G_{F}=2 \int_{0}^{\delta} \int_{z}^{\delta} \int_{0}^{\cos ^{-1}(z / \xi)}\left(c(\xi) s_{0}^{2} \xi / 2\right) \xi d \theta d \xi d z
$$

where, $c(\xi)$ denotes the micro-elastic modulus [2] and $\mathrm{z}$ denotes the distance between the point $\mathrm{x}$ and the crack surface as shown in Fig. 2, the $s_{c}$ and $s_{t}$ are computed as follows[4]:

$$
s_{c}=-\frac{f_{c}}{E}, s_{t}=\frac{f_{t}}{E}
$$

The following equation was used to define $G_{F}$ for tensile concrete [9]:

$$
G_{F}=\left(0.0469 d_{\max }^{2}-0.5 d_{\max }+26\right)\left(\frac{f_{c}^{\prime}}{10}\right)^{0.7}(\mathrm{~N} / \mathrm{m})
$$

where, $d_{\max }(\mathrm{mm})$ is the maximum coarse aggregate size, it is usually taken as $40 \mathrm{~mm}$, and $f_{c}^{\prime}$ (Mpa) is the cylinder compressive strength of concrete. After substituting (3) into (1), the value of the breaking critical stretch $s_{0}$ can be obtained at last.

\section{Discretization and Numerical Implementation}

As a usual, the peridynamic equations of motion could be solved by utilizing a numerical approximation method involves the discretization of the reference configuration into particles with a certain volume, thus, The integrals can be denoted by the finites sums:

$$
\rho \ddot{\mathbf{u}}_{n}^{i}=\sum_{j=1}^{N_{i}} \mathbf{f}\left(\boldsymbol{\eta}_{t_{n}}, \xi\right) V_{j}+\mathbf{b}_{t_{n}}^{i}
$$

where, $\ddot{\mathbf{u}}_{n}^{i}$ denotes the acceleration of the point $x_{i}$ at time $t_{n}, N_{i}$ is the total number of particles within the horizon of the point $x_{i}, \mathbf{f}\left(\mathbf{\eta}_{t_{n}}, \boldsymbol{\xi}\right)$ and $\mathbf{b}_{t_{n}}^{i}$ is the pairwise force and the body force at time $t_{n}$, respectively. In order to apply the peridynamic equations of motion to solve quasi-static 
problems, Kilic and Madenci [7] introducing an artificial damping to guide the solution to approach the steady state and using the Adaptive Dynamic Relaxation (ADR) scheme proposed by Underwood [8] to determine the most effective damping coefficient at each time step n, as follows:

$$
\ddot{\mathbf{u}}_{n}^{i}+c_{n} \dot{\mathbf{u}}_{n}^{i}=\mathbf{F}_{n}^{i} / \lambda_{i}
$$

where, $\mathbf{F}_{n}^{i}$ is the resultant force density vector, $c_{n}$ and $\lambda_{i}$ is the damping coefficient at the $n^{\text {th }}$ iteration and the modified density at the point $x_{i}$,expressed as:

$$
\begin{aligned}
& c_{n}=2 \sqrt{\left(\mathbf{u}_{n}^{i}\right)^{T 1} \mathbf{K}_{n}^{i i}\left(\mathbf{u}_{n}^{i}\right) /\left(\left(\mathbf{u}_{n}^{i}\right)^{T}\left(\mathbf{u}_{n}^{i}\right)\right)} \\
& \lambda_{i} \geq \frac{1}{4} \Delta t^{2} \sum_{j=1}^{N_{i}}|\xi \cdot \mathbf{e}| \cdot \mathbf{c}(\xi) /|\xi|^{2}
\end{aligned}
$$

In which $\Delta t$ is the time step size, $\mathbf{e}$ is a unit vector along the $\mathbf{x}-, \mathbf{y}^{-}$, or $\mathrm{z}$-direction, and ${ }^{1} \mathbf{K}_{n}^{i i}$ is the diagonal stiffness matrix of the system, given as:

$$
{ }^{1} K_{n}^{i i}=-\left(\mathbf{F}_{n}^{i} / \lambda_{i}-\mathbf{F}_{n-1}^{i} / \lambda_{i}\right) /\left(\Delta t \dot{u}_{n-1 / 2}^{i}\right)
$$

Finally, with the assumptions that $\mathbf{u}_{0}^{i} \neq 0$ and $\dot{\mathbf{u}}_{0}^{i}=0$, velocities and displacements at point $x_{i}$ for the next time step can be obtained by central difference explicit integration as

$$
\begin{aligned}
& \dot{\mathbf{u}}_{1 / 2}^{i}=\Delta t \mathbf{F}_{0}^{i} /\left(2 \lambda_{i}\right) \\
& \dot{\mathbf{u}}_{n+1 / 2}^{i}=\left[\left(2-c_{n} \Delta t\right) \dot{\mathbf{u}}_{n-1 / 2}^{i}+2 \Delta t \mathbf{F}_{n}^{i} / \lambda_{i}\right] /\left(2+c_{n} \Delta t\right) \\
& \mathbf{u}_{n+1}^{i}=\mathbf{u}_{n}^{i}+\Delta t \dot{\mathbf{u}}_{n+1 / 2}^{i}
\end{aligned}
$$

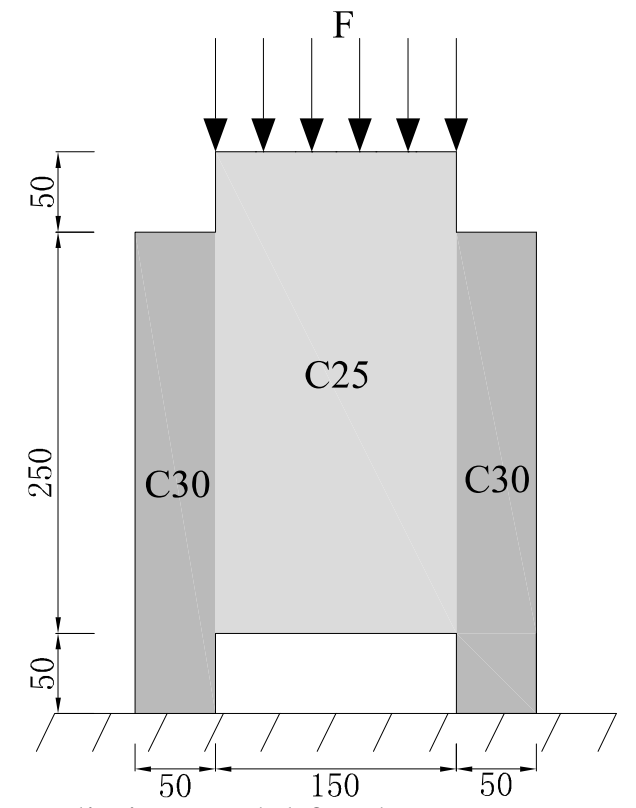

Fig. 3 Prediction model for the concrete specimen.

\section{Progressive Damages of the Underpinned Concrete Column}

Although the mechanical behavior of deep concrete beams has been a subject of many experimental studies since the 1960s, and researchers have proposed different empirical formulas and analytical models for the deep concrete beams, the accurate prediction of failure mechanism and bearing strength of deep concrete beam remains difficult, $\mathrm{Bi}$ et al. [9] have made an experiment of underpinned concrete column subjected to a compressive loading and put forward a bearing 
capacity formula, To further demonstrate the capabilities of the proposed peridynamic approach and concrete constitutive model, In this section, we simulate the progressive process of damages and failures of the underpinned concrete column. Fig. 3 shows the corresponding geometrical features of the concrete specimen, and the material properties are listed in Table 1.

Table 1. Material parameters used in the simulation.

\begin{tabular}{|l|l|l|}
\hline Concrete type & C25 & C30 \\
\hline Young's modulus E $(\mathrm{MPa})$ & 28000 & 30000 \\
\hline Uniaxial tensile strength $f_{t}(\mathrm{MPa})$ & 2.01 & 3.41 \\
\hline Uniaxial compressive strength $f_{c}(\mathrm{MPa})$ & 25.71 & 31.22 \\
\hline cylinder compressive strength $f_{c}^{\prime}(\mathrm{MPa})$ & 20.31 & 24.65 \\
\hline fracture energy $G_{F}(\mathrm{~N} / \mathrm{m})$ & 130.5 & 148.3 \\
\hline maximum coarse aggregate size $d_{\max }(\mathrm{mm})$ & 40 & 40 \\
\hline tensile stretch limit $s_{t}$ & $7.179 \times 10^{-5}$ & $8.033 \times 10^{-5}$ \\
\hline compressive stretch limit $s_{c}$ & $9.182 \times 10^{-4}$ & $1.041 \times 10^{-3}$ \\
\hline critical stretch $s_{0}$ & $5.704 \times 10^{-4}$ & $5.874 \times 10^{-4}$ \\
\hline
\end{tabular}

Similarly, the underpinned concrete column is discretized into particles uniformly, so that all particles would have equivalent volumes. The prediction model had a constant in-plane grid spacing of $5 \mathrm{~mm}$ and a constant material horizon $\delta=20 \mathrm{~mm}$ (four times the grid spacing). The compressive loading was applied on the top of the column step by step with an increment of $10 \mathrm{kN}$ which keep the same as in [9]. A new equilibrium state can be reached in each step until the system become instable, Fig. 10 illustrates the process of progressive damage in the underpinned concrete column under monotonic compression, and the deformation of the column has here been magnified 1000 times. As the load increases, obvious bending and shear deformation can be observed in the prediction model, and when the external load increases to $163 \mathrm{kN}$, the accumulation of damages leads to irreversibly broken in the bonds around the middle of the bottom edge of the column (as shown in Fig. 4(b)), However, the structure can still resist the increasing external load until the ultimate load $(205 \mathrm{kN})$ is reached, after which the crack started to spread rapidly along the central axis (see Fig. 4(d)).

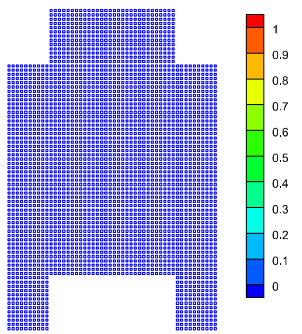

(a) $\mathrm{F}=0 \mathrm{KN}$

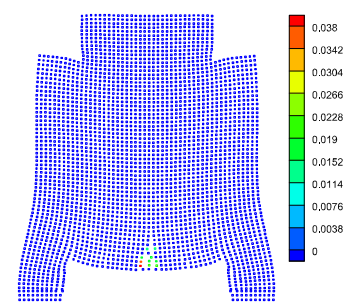

(c) $\mathbf{F}=205 \mathrm{KN}$

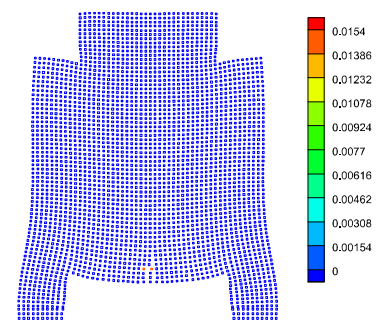

(b) $\mathrm{F}=163 \mathrm{KN}$

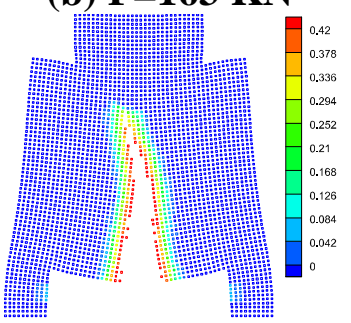

(d) 5000 iterations after $\mathbf{F}=\mathbf{2 0 5} \mathrm{KN}$

Fig. 4 The progressive failure of the underpinned concrete column.

Finally, from the simulation results of the underpinned concrete column, high agreement of the crack propagation path could be seen between the peridynamic model and the experimental 
specimen, as indicated in Fig. 5. Moreover, the crack-resisting load $(163 \mathrm{kN})$ and ultimate load $(205 \mathrm{kN})$ agreed fairly well with the experimental results $180 \mathrm{kN}$ and $220 \mathrm{kN}$, respectively. It is denoted that this peridynamic approach was able to deal with discontinuous problems with enough accuracy.
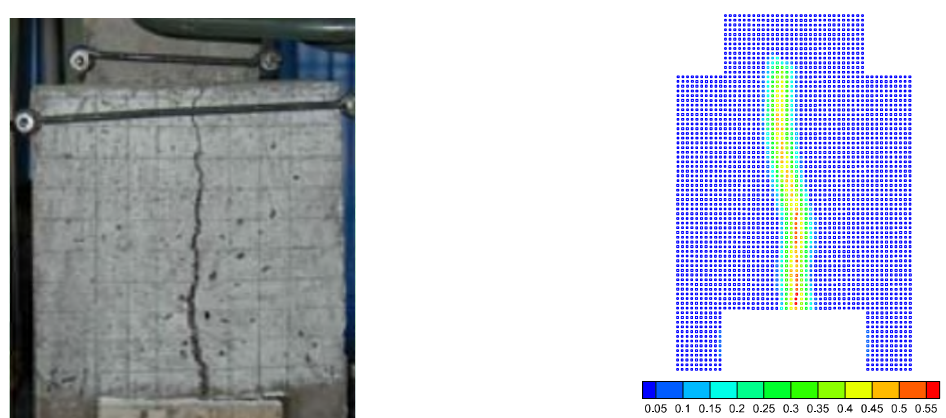

Fig. 5 Comparison of final failure modes between peridynamic and experimental results.

\section{Conclusion}

In this paper, an improved peridynamic model for concrete structures had been developed, and the investigation on the capabilities of the proposed numerical model to reproduce the progressive failure process of materials and structures under monotonic loading was also presented. The extreme failure load and the final failure mode of the underpinned concrete column by analysis of peridynamic approach match well with that of the experimental observations.

\section{References}

[1] E. Madenci, E. Oterkus. Peridynamic theory and its applications. New York: Springer-Verlag, 2014.

[2] S. A. Silling. Reformulation of elasticity theory for discontinuities and long-range forces, J. Mech. Phys. Solids, 48 (2000) 175-209.

[3] S. A. Silling, E. Askari. A meshfree method based on the peridynamic model of solid mechanics, Comput. Struct. 83 (2005) 1526-1535.

[4] W. Gerstle, N. Sau, E. Aguilera. Micropolar peridynamic modeling of concrete structures, Proceedings of the $6^{\text {th }}$ International Conference on Fracture Mechanics of Concrete and Concrete Structures, Toronto, Canada, June 2007, pp. 475-481.

[5] D. Huang, G. Lu, C. Wang, et al. An extended peridynamic approach for deformation and fracture analysis, Eng. Fract. Mech. 141 (2015) 196-211.

[6] Z. P. Bažant, E. Becq Giraudon. Statistical prediction of fracture parameters of concrete and implications for choice of testing standard, Cem. Concr. Res. 32 (2002) 529 -556.

[7] B. Kilic, E. Madenci. An adaptive dynamic relaxation method for quasi-static simulations using the peridynamic theory, Theoret. Appl. Fract. Mech. 53 (2010)194-204.

[8] P. Underwood. Dynamic relaxation in Computational Methods for Transient Analysis, Amsterdam: New-Holland, 1983, pp. 245-265.

[9] Z. Bi, Q. Wang, X. He. Study on bearing capacity test on plain concrete underpinned column, J. Liaoning Technical University, 24 (2005) 692-694. 